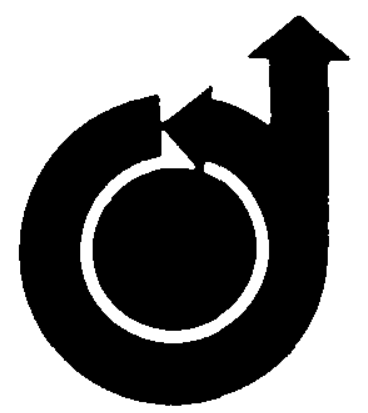

79-0288

\title{
Recent Experiments on Heterogeneous Detonation Waves
}

J.A. Nicholls, R. Bar-Or, Z. Gabrijel and E. Petkus, University of Michigan, Ann Arbor, Mich.

\section{7th AEROSPACE SCIENCES MEETING}

New Orleans, La./January 15-17, 1979

For permission to copy or republish, contact the American Instltute of Aeronautics and Astronautics, 1290 Avenue of the Amerlcas, New York, N.Y. 10019. 


\section{J.A. Nicholls*, R. Bar-Or**, Z. Gabrijel ${ }^{\dagger}$, E. Petkus ${ }^{\dagger+}$}

The University of Michigan, Ann Arbor, MI

\section{Abstract}

Some recent results on the blast wave initiation of cylindrical heterogeneous detonation waves are presented herein. Results include a comparison of the rate of decay of the cylindrical blast wave through nitrogen alone with that wherein 400 um liquid drops are dispersed throughout the nitrogen. Heterogeneous reactive mixture results include kerosene-air, kerosene-oxygen, kerosene + NPN (normal propyl nitrate)-air, kerosene + NPN-oxygen, heptane-air, and heptane-oxygen. Some results are presented wherein the inner portion of the cloud is devoid of fuel but the decaying blast wave produces detonation later. A few high speed framing camera photographs of cylindrical heterogeneous detonation waves are shown.

\section{Introduction}

This paper presents some recent results on an ongoing study of the blast wave initiation and propagation of cylindrical heterogeneous detonation waves. Particular aspects of interest in this study are the details of and the initiator energy required for the initiation of detonation, the characteristics of the wave propagation, the influence of physical and chemical properties of the fue1, and wave propagation through a cloud which is non-uniform in fuel-oxidizer ratio.

The experimental facility employed is essentially the same as that described earlier ${ }^{1,2}$ so that only a cursory description will be given here. The sectored shock tube, shown in Fig. 1 , is designed to model a sector of a cylindrical combustible cloud. The angle of the sector is $20^{\circ}$, the "height" of the cloud (distance between the side walls) is $5.2 \mathrm{~cm}$, and the radius is about $140 \mathrm{~cm}$. The fuel drops are dispersed throughout the gaseous oxidizer by flowing the liquid fuel through as many as 322 needles and pulsing this flow at about the Rayleigh frequency. With the current size of needles in use, the resultant uniform drop size is about $400 \mu \mathrm{m}$. The cylindrical blast wave is formed by firing a blasting cap (Dupont E-106) and a measured amount of condensed explosive (Dupont Detasheet $\mathrm{C}$ ). The propagation of the wave in the radial direction is monitored by 14 time of arrival pressure switches. This position-time data can then be converted to velocity versus radius information. For photographic work, special side plates are used for the initial part of the chamber which

*Professor, Aerospace Engineering Department; Associate Fellow, AIAA.

**Doctoral Candidate.

tDoctoral Candidate.

t'Student; Student Member, AIAA. incorporate windows on the centerline which are centered about a radius of about $65 \mathrm{~cm}$. The windows are approximately $2 \mathrm{~cm}$ by $10 \mathrm{~cm}$ in the radial direction and $2.5 \mathrm{~cm}$ thick. A Beckman-Whitley 330 camera was used which can yield 80 frames at up to 2 million frames per second with about $1 / 3 \mu \mathrm{s}$ exposure time for each frame. The camera was used at a speed of about 500,000 frames per second. A parallel beam light source was triggered by a pressure switch and double exposure was avoided by pulsing the xenon light source for a limited amount of time.

The chamber was operated with 42 rows with seven needles $(0.02 \mathrm{~cm}$ I.D.) per row. For the oxygen case, the fuel flow was maintained constant and the air replaced by oxygen. As a consequence, the mixture ratio was quite lean in the oxygen studies. The resulting equivalence ratios and the expected Chapman-Jouguet detonation velocities, as calculated by the Gordon-McBride program ${ }^{3}$, are given in the table below. In calculating $\mathrm{DCJ}$ the fuels were assumed to be in the gaseous phase but the enthalpy of formation used was that for the liquid.

\begin{tabular}{|c|c|c|c|c|}
\hline exidizer & & AIR & \multicolumn{2}{|c|}{ OXYGEN } \\
\hline Fuel & $\phi$ & $\mathrm{D}_{\mathrm{CJ}}(\mathrm{m} / \mathrm{sec}$ & $\phi$ & $\mathrm{D}_{\mathrm{Cu}}(\mathrm{m} / \mathrm{sec}$ \\
\hline Kerosene & 1.6 & 1810 & 0.33 & 1876 \\
\hline $\begin{array}{l}75 \% \text { Kerosene } \\
25 \% \text { NPN }\end{array}$ & 1.38 & 1850 & 0.28 & 1812 \\
\hline Heptane & 1.45 & 1814 & 0.3 & 1840 \\
\hline
\end{tabular}

Recently obtained experimental results will now be presented and discussed.

\section{Experimental ResuIts}

A series of runs was conducted which shows the weakening effect of the drops on the blast wave. Figure 2 shows radius-time results for three separate runs wherein 5 grams of explosive (plus the

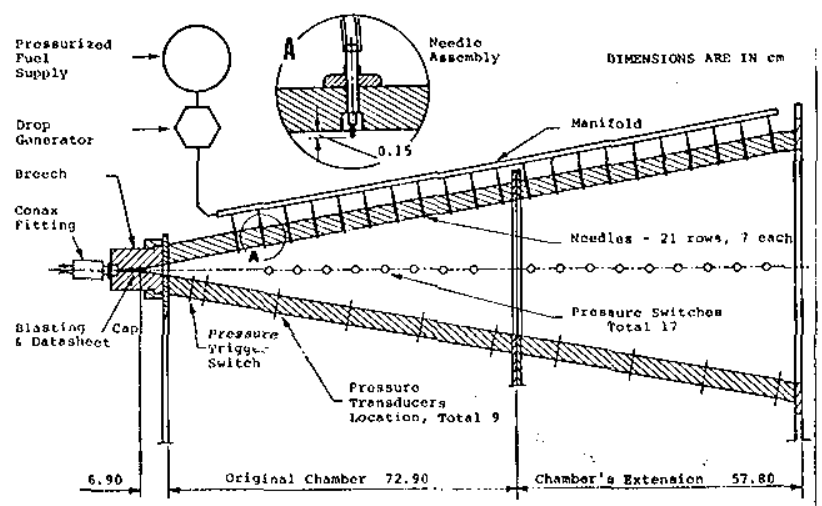

Figure 1. Schematic of Experimental Set-up. 


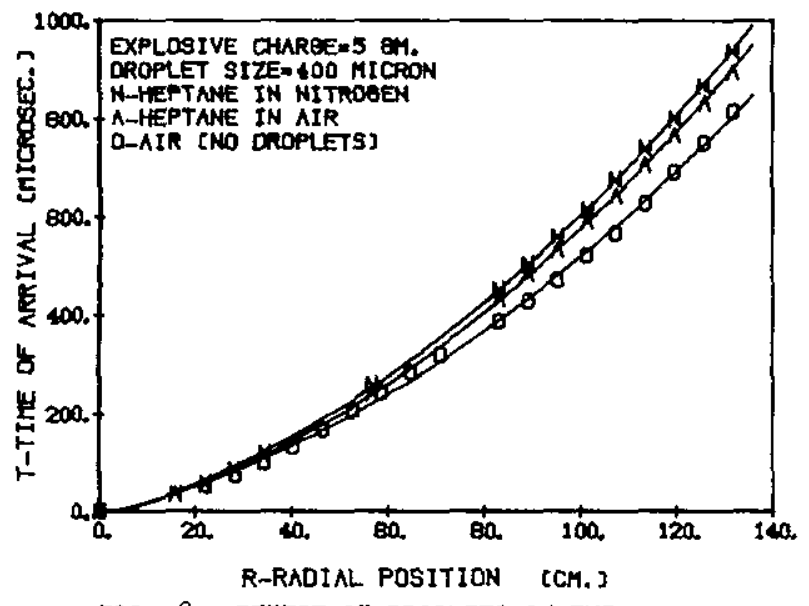

FIB. 2. EFFECT OF DROPLETS ON THE DECAY OF BLAST YAVES

blasting cap) were used in each case. The lowest curve, which represents the highest wave velocity, corresponds to a cylindrical blast wave in air with no fuel present. The upper, or slowest velocity curve, is a repeat except that heptane drops were present and nitrogen replaced the air. The marked reduction in wave velocity by the drops is readily apparent. The third curve corresponds to the same strength blast wave into heptane drops and air. The wave velocity is increased somewhat over that in the nitrogen case, thus indicating that combustion is partially supporting the wave. However, detonation does not occur and the wave speed is well below that for the pure blast wave case.

Figure 3 represents results for various run conditions for a constant initiator energy level. As can be seen, the blast wave in air is somewhat faster than the reacting blast wave (no detonation) in kerosene and air. The observed velocity in kerosene-oxygen is $1600 \mathrm{~m} / \mathrm{sec}$ as compared to the theoretical value of $1876 \mathrm{~m} / \mathrm{sec}$. This lower detonation velocity is believed to be attributable to losses to the chamber walls. Some further experimental results in kerosene-oxygen for different energy levels are shown in Fig. 4. It can be noted that the higher energy levels produce stronger blast waves which decay to a constant velocity, presumably the detonation velocity. Curves 2 and 3 are close to the same velocity and curve 1 is

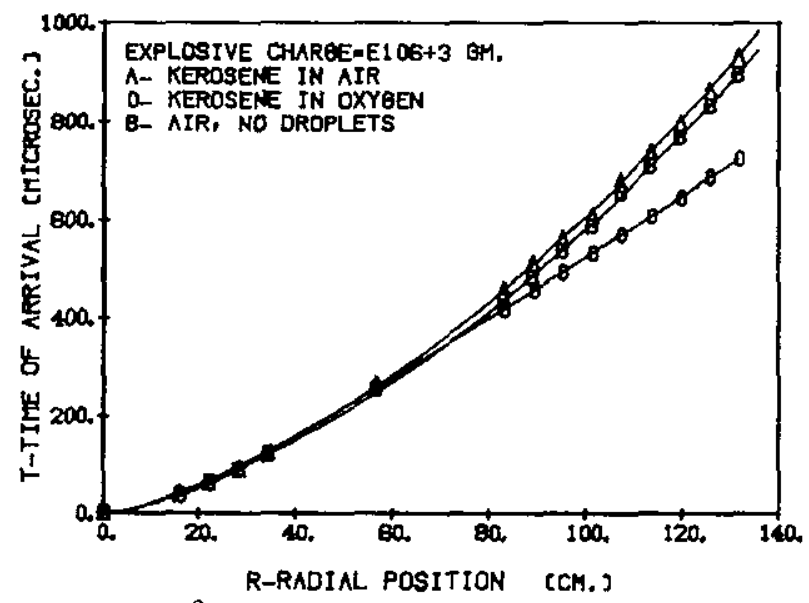

FIB. 3. BLAST AND DETONATION WAVES

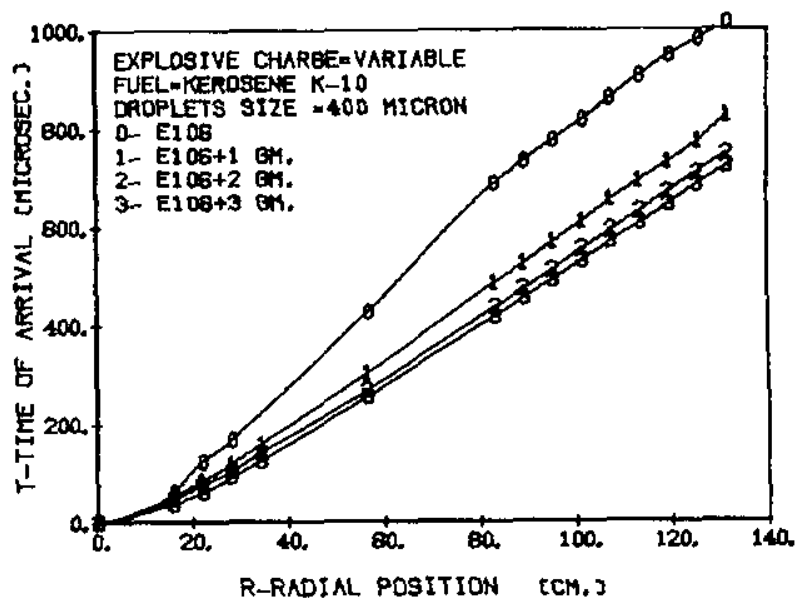

FIG. 4. DETONATIONS IN KEROSENE/OXYGEN

slightly slower. In the case of the blasting cap alone, acceleration to detonation velocity occurs only in the second half of the chamber and the final velocity seems to be the same as for the larger charges.

Further experiments were conducted wherein the kerosene was sensitized by the addition of $25 \%$ (by volume) of normal propyl nitrate (NPN). The results are shown in Fig. 5, wherein the energy leve1 was the same for every run. Two curves for the nonsensitized case, shown earlier, are included for comparison. Two separate runs are shown for sensi. tized kerosene-air. In one case the smooth curve shown indicates constant velocity, or detonation. However, the indicated velocity, $1350 \mathrm{~m} / \mathrm{sec}$, is low and there is some doubt that detonation occurs. The open circles for the other run, not connected by a curve, are above the curve except at about $115 \mathrm{~cm}$ a rapid acceleration of the wave is indicated It is concluded that this kerosene-NPN-air mixture is close to detonation for this energy level and that higher initiator energies would produce detonation. Finally, the sensitized kerosene-oxygen mixture is seen to accelerate more rapidly to detonation conditions than in the unsensitized case. The observed velocity of $1660 \mathrm{~m} / \mathrm{sec}$ is somewhat lower than the theoretical one $(1812 \mathrm{~m} / \mathrm{sec})$ and slightly higher than the non-sensitized case.

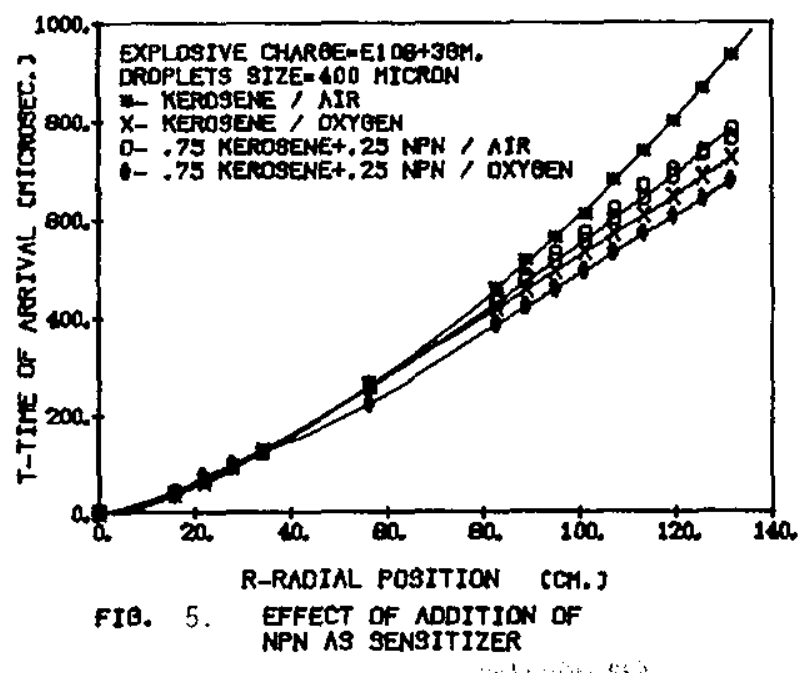


Some experimental results for blast and detonation waves in heptane-air and heptane-oxygen mixtures are shown in Fig. 6 . It is obvious that the high energy run ( 5 grams) for heptane-air did not result in detonation. On the other hand, a detonation was produced almost immediately for the heptaneoxygen case with only a blasting cap. The higher energy run (1 gram) produced essentially the same velocity after the blast wave decayed.

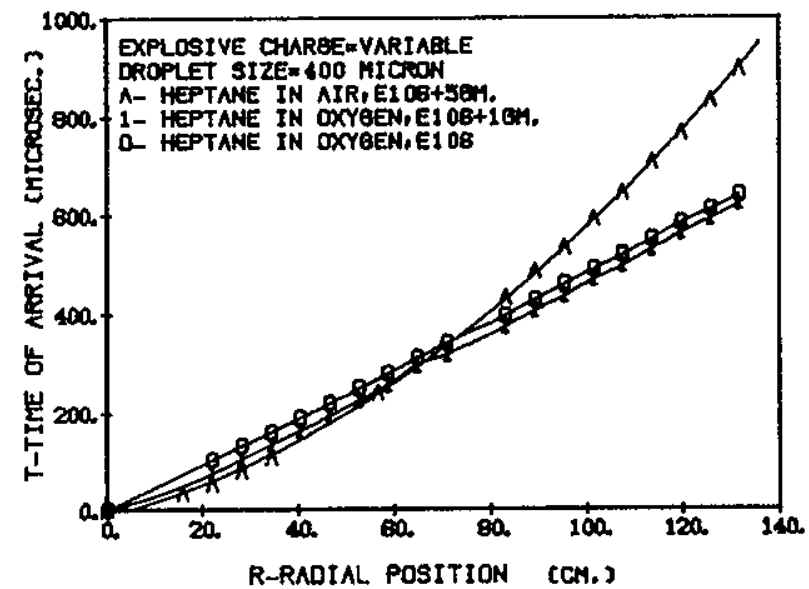

FI8,6 ELAST AND DETONUTION YAVES IN HEPTNE/AIR AND HEPTANE/OXYBEN HIXTURES

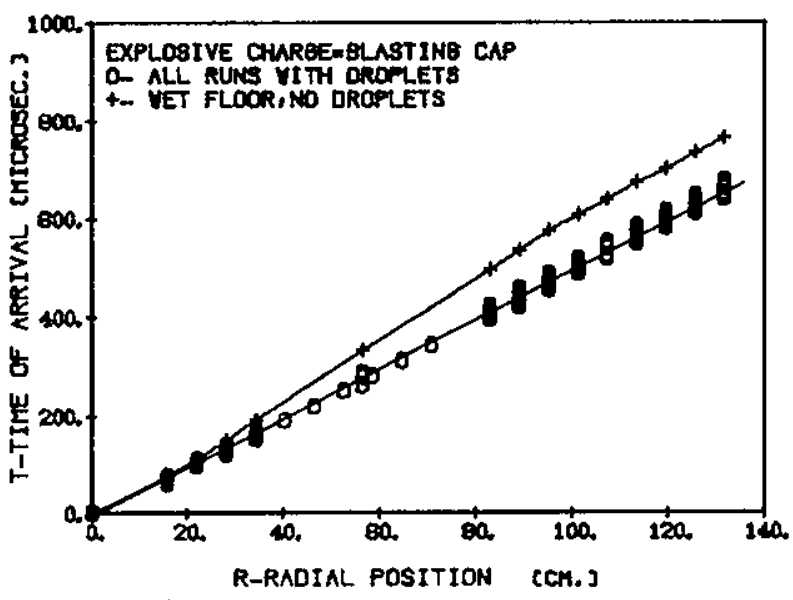

FIB. 7. DETONATIONS IN HEPTANEJOXYOEN MIXTURES

Additional heptane-oxygen runs are shown in Fig. 7. The lower constant velocity detonation curve is the average of many experiments and the measured velocity is $2000 \mathrm{~m} / \mathrm{sec}$. For the upper curve, the drops were not being produced. However, there was some dripping from the needles and the bottom wall was quite wet with fuel. After some decay, it appears that detonation was established. Apparently the high vapor pressure of heptane is leading to an appreciable vapor content. Considering the vapor pressure of heptane, we could expect a $\phi=0.5$ to 0.6 in the vapor phase. Calculation of the $\mathrm{CJ}$ velocity for these conditions indicates values of $2037 \mathrm{~m} / \mathrm{sec}$ and $2112 \mathrm{~m} / \mathrm{sec}$, respectively, which are in excellent agreement with our measured value.

A comparison of various fuels with oxygen is shown in Fig. 8 for the lowest initiator energy level. There appears to be a distinct separation in the reactivities of the various combinations.

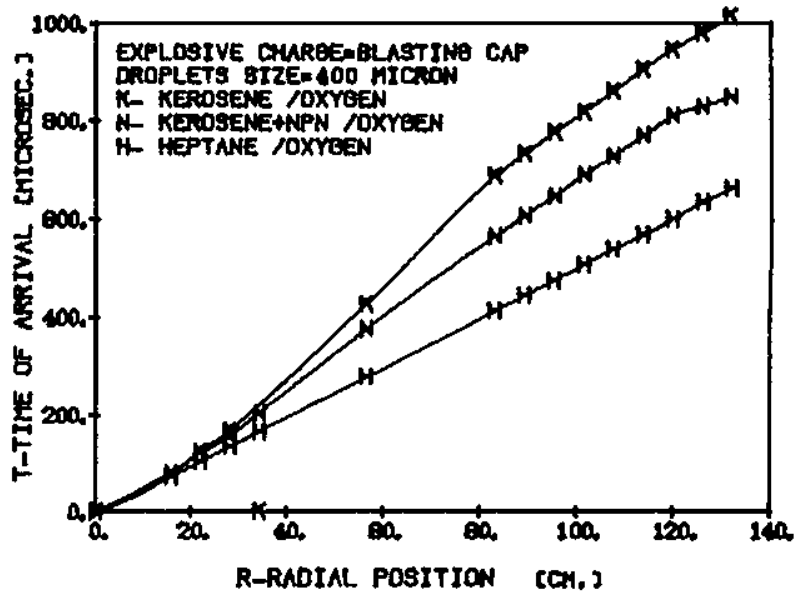

FIO. 8. DETONATIONS IN DIFFERENT FUELS

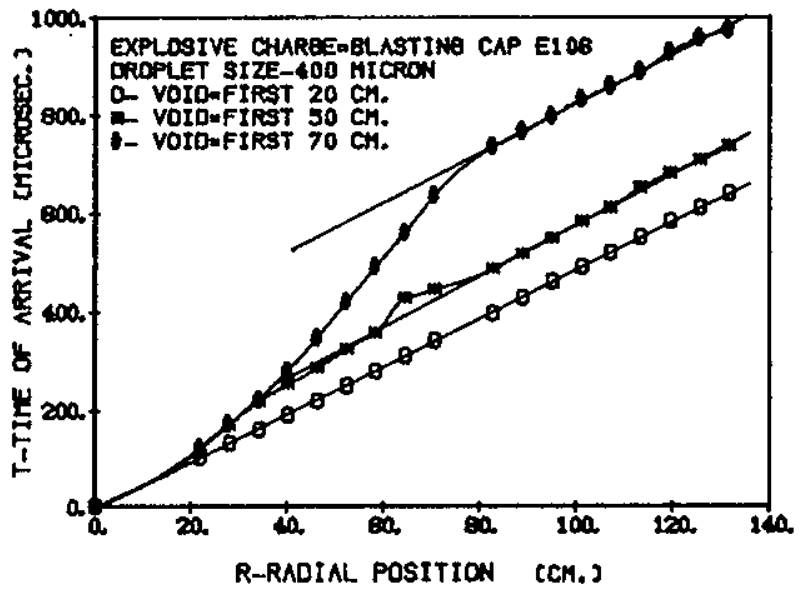

FI6. 9. EFFECT OF VOIDS ON DETONATIONS OF HEPTANE/OXYGENE

While detonation in heptane-oxygen is initiated right away, the blast wave in both kerosene-oxygen and kerosene + NPN-oxygen is decaying much more before accelerating to a detonation wave. The rapid acceleration that appears at large radius for kerosene + NPN and, to some extent, for kerosene indicates that stable propagation has not yet been attained.

The effect of regions of various extent which are devoid of fuel and at the cloud center is shown in Fig. 9. For the case of no fuel up to a radius of $20 \mathrm{~cm}$, detonation is established almost immediately. For the $50 \mathrm{~cm}$ case the reacting blast wave decays but then goes over to detonation. The transition is somewhat upstream of the $50 \mathrm{~cm}$ position and this may be attributable to the diffusion of vapors upstream. Similar results are seen for the $70 \mathrm{~cm}$ case. The detonation velocities are almost the same in each case.

A few frames from a high speed framing camera record of a cylindrical heptane-oxygen detonation are shown in Fig. 10. The framing speed was approximately 500,000 frames per second and the time between the frames shown in the figure is 8.1 $\mu \mathrm{sec}$. The reference wire system is $5 \mathrm{~cm}$ apart. In the upper frame, three vertical arrays of droplets can be seen; the drop producing stations are about $2.5 \mathrm{~cm}$ apart. In the upper photograph, the shock wave (not very sharp) is to the left of the double 

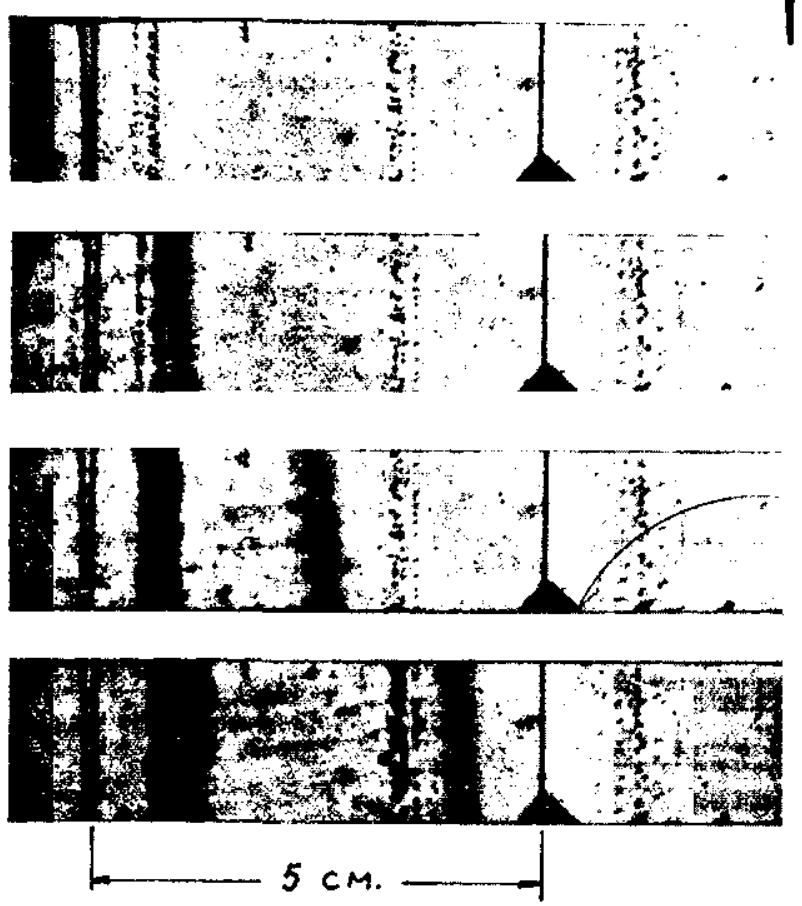

Figure 10. Detonation Wave in Two Phase Heptane-0xygen Mixture

wire and moving to the right. In the second photograph the shock has passed over the drops from the 7 needles and some distortion of the drops is apparent. In the third and fourth photographs considerable aerodynamic shattering of the drops has resulted but it is not obvious as to whether ignition has occurred yet. In the last photograph the shock has traversed the second column of drops. There appears to be horizontal layers behind the shock but an explanation is not in hand.

\section{Concluding Remarks}

As brought out in the discussion of the experimental results, many liquid fuels in air lead to difficulties as far as the establishment of detonation is concerned. As a consequence, it is often difficult to tell from the laboratory scale tests whether detonation is achieved or not. Further, there is the strong possibility that the chamber gives some confinement effects for those reactions with long reaction zones. Accordingly, it is planned to use oxygen enrichment in order to achieve easier and faster initiation of detonation. This aspect is of great importance when studying the propagation rate through a non-uniform mixture. Another aspect of importance is the vapor pressure of the liquid. While a liquid with relatively high vapor pressure, such as heptane, is certainly of interest, $4 \mathrm{t}$ would be revealing to ga in controlled information for those cases where the vapor phase of the fuel would not be expected to enter strongly. Further studies will follow this path, at least in part.

\section{ACKNOWL.EDGMENT}

This research is supported by the U.S. Army Research Office under Grants DAAG29-77-G-0104 and DAAG-29-78-G-0116.

\section{REFERENCES}

1. Gabrijel, Z. and Nicholls, J.A., "Cylindrical Heterogeneous Detonation Waves, "presented at the Sixth International Colloquium on Gas Dynamics of Explosions and Reactive Systems, Stockholm, Sweden, August 1977, to be pub] ished in Acta Astronautica.

2. Nicholls, J.A., Sichel, M., Gabrijel, Z., Oza, R., and Vander Molen, R., "Detonability of Unconfined Natural Gas-Air Clouds," presented at the Seventeenth International Symposium on Combustion, University of Leeds, England, August 20-25, 1978; accepted for publication in the proceedings.

3. Gordon, S. and McBride, B.J., "Computer Program for Calculation of Complex Chemical Equilibrium Compositions, Rocket Performance, Incident and Reflected Shocks, and Chapman-Jouguet Detonation," NASA SP-273, 1971. 